\title{
Dying in their prime: determinants and space-time risk of adult mortality in rural South Africa
}

\author{
Benn Sartorius ${ }^{1,2}$, Kathleen Kahn ${ }^{2,3,4}$, Mark A. Collinson ${ }^{2,3,4}$, Kurt Sartorius ${ }^{5}$, Stephen M. \\ Tollman ${ }^{2,3,4}$ \\ ${ }^{1}$ Division of Epidemiology and Biostatistics, School of Public Health, Faculty of Health Sciences, University of \\ the Witwatersrand, Johannesburg, South Africa; ${ }^{2} M R C /$ Wits Rural Public Health and Health Transitions \\ Research Unit (Agincourt), School of Public Health, Faculty of Health Sciences, University of the \\ Witwatersrand, Johannesburg, South Africa; ${ }^{3}$ Centre for Global Health Research, Epidemiology and Global

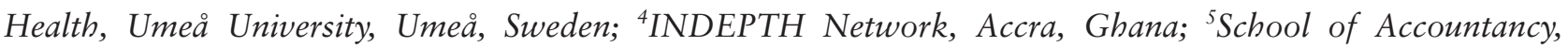 \\ Faculty of Commerce, Law and Management, University of the Witwatersrand, Johannesburg, South Africa
}

\begin{abstract}
A longitudinal dataset was used to investigate adult mortality in rural South Africa in order to determine location, trends, high impact determinants and policy implications. Adult (15-59 years) mortality data for the period 1993-2010 were extracted from the health and demographic surveillance system in the rural sub-district of Agincourt. A Bayesian geostatistical frailty survival model was used to quantify significant associations between adult mortality and various multilevel (individual, household and community) variables. It was found that adult mortality significantly increased over time with a reduction observed late in the study period. Non-communicable disease mortality appeared to increase and decrease in parallel with communicable mortality, whilst deaths due to external causes remained constant. Male gender, unemployment, circular (labour) migrant status, age and gender of household heads, partner and/or other household death, low education and low household socio-economic status were identified as significant and highly attributable determinants of adult mortality. Health facility remoteness was a risk for adult mortality and households falling outside a critical buffering zone were identified. Spatial foci of higher adult mortality risk were observed, indicating a strong non-random pattern. Communicable diseases differed from non-communicable diseases with respect to spatial distribution of mortality. Areas with significant excess mortality risk (hot spots) were found to be part of a complex interaction of highly attributable factors that continues to drive differential space-time risk patterns of communicable (HIV/AIDS and tuberculosis) mortality in Agincourt. The impact of HIV mortality and its subsequent lowering due to the introduction of antiretroviral therapy was found to be clearly evident in this rural population.
\end{abstract}

Keywords: adult mortality, spatial risk, survival modelling, Bayesian inference, determinants, attributable fractions, health and demographic surveillance, South Africa.

\section{Introduction}

Adult mortality in many developing countries has received scant attention in recent decades in contrast to childhood mortality (Murray and Feachem, 1990). This lack of focus is especially surprising in subSaharan Africa (SSA) where the risk of dying before 60 years of age remains excessively high (Murray and Feachem, 1990). Despite a worldwide reduction in communicable disease (Murray and Lopez, 1997), high levels of communicable disease (HIV/AIDS) have

Corresponding author:

Benn Sartorius

School of Public Health

Faculty of Health Sciences - University of the Witwatersrand 27 St Andrews Road, Parktown, Johannesburg, South Africa Tel. +27 11717 2313; Fax +27 117172084

E-mail: benn.sartorius@wits.ac.za drastically reduced life expectancy in SSA and South Africa. This has slowed the expected health transition where communicable disease gives way to non-communicable disease as a result of progress in health care as well as social and economic development (Preston and Nelson, 1974). Simultaneously, many parts of SSA are experiencing an upsurge in non-communicable disease, such as cancer and cardiovascular disease, that is projected to increase until 2020 (Murray and Lopez, 1997), largely due to lifestyle-related factors such as obesity, smoking and alcohol consumption (WHO, 2002). This projected upsurge is particularly important because of its impact on economically productive adults (WHO, 2008), who are responsible for the welfare of younger and older age groups (Feachem et al., 1992). Thus, adult mortality in SSA presents a changing set of dynamics challenging the limited healthcare resources of the region (Murray and Feachem, 1990) because of its impact on the availability and produc- 
tivity of working adults (Schatz and Ogenmefun, 2007; Sartorius et al., 2011b).

Adult mortality is a vital indicator for planning health care interventions, but data are often lacking in SSA (Kaufman et al., 1997). Health and demographic surveillance systems (HDSS), though not representative at the national level, are often the only means to assess and better understand population levels and trends and allow the assessment of cause-specific mortality on a longitudinal basis (Kaufman et al., 1997; Kuh and Shlomo, 2004). Adult mortality can be influenced by a combination of variables at the individual, household and community level. A conceptual framework (Fig. 1) was developed to guide and interpret the analysis of adult mortality determinants. The framework expands previous work to include the effect of historical legacies, as well as account for a spatial temporal aspect (Victora et al., 1997).

Individuals that emerge from households are unique biologically (and differentially frail ${ }^{1}$ ) but are also a product of their surrounding environment, including their ethnic norms, experience and behaviour. Historical legacies have a pervasive long-term effect on the cultural dimensions of a society, as well as the location and efficiency of its institutions (Hofstede, 1986; Williamson, 2000). In South Africa, household characteristics have been shaped by a $100+$ years of colonial and apartheid rule and have had a profound impact on the location of infrastructure and forced

${ }^{1}$ Unobserved susceptibility to death (frailty) is a concern in survival analyses, where individual survival probability variations cannot be ignored. ethnic settlement in geographically defined areas (Bryceson, 2004; Mertz et al., 2005; Sherbinin et al., 2008). This has led to differential education and health care quality (Williamson, 2000) and impacted the socio-economic status (SES) of many households (Machethe, 2004). Household characteristics that have been influenced over the medium term include household size, access to services, ethnicity, physical location, employment, health, access to land and education (Schwarze and Zeller, 2005; Vermeulen et al., 2008; Xiangxing et al., 2008). Individual characteristics that can influence adult behaviour and lifestyle choices include age, gender, nationality, religion, education (intelligence), income and partner outcome (direct and indirect consequences). Moreover, a combination of these individual attributes, determines the likelihood of disease type and the risk it poses with respect to their mortality (Victora et al., 1997; Brown et al., 2004). Finally, exogenous shocks like drought, famine or disease (e.g. HIV/AIDS), have had a major influence on mortality in SSA (Hosegood et al., 2004; Kahn et al., 2007).

Given the projected burden of disease in SSA and associated socio-economic impact, this paper investigates the dynamics, trends, causality and implications of adult mortality in rural South Africa. It builds on previous work in this setting by (i) assessing neglected adult mortality within a detailed conceptual framework; (ii) introducing population attributable fractions to quantify determinant impact for policy makers; (iii) analysing the importance and potential emergence of non-communicable adult mortality as a result of lifestyle and nutritional transitions; (iv) using a more complex analytical framework (continuous time-

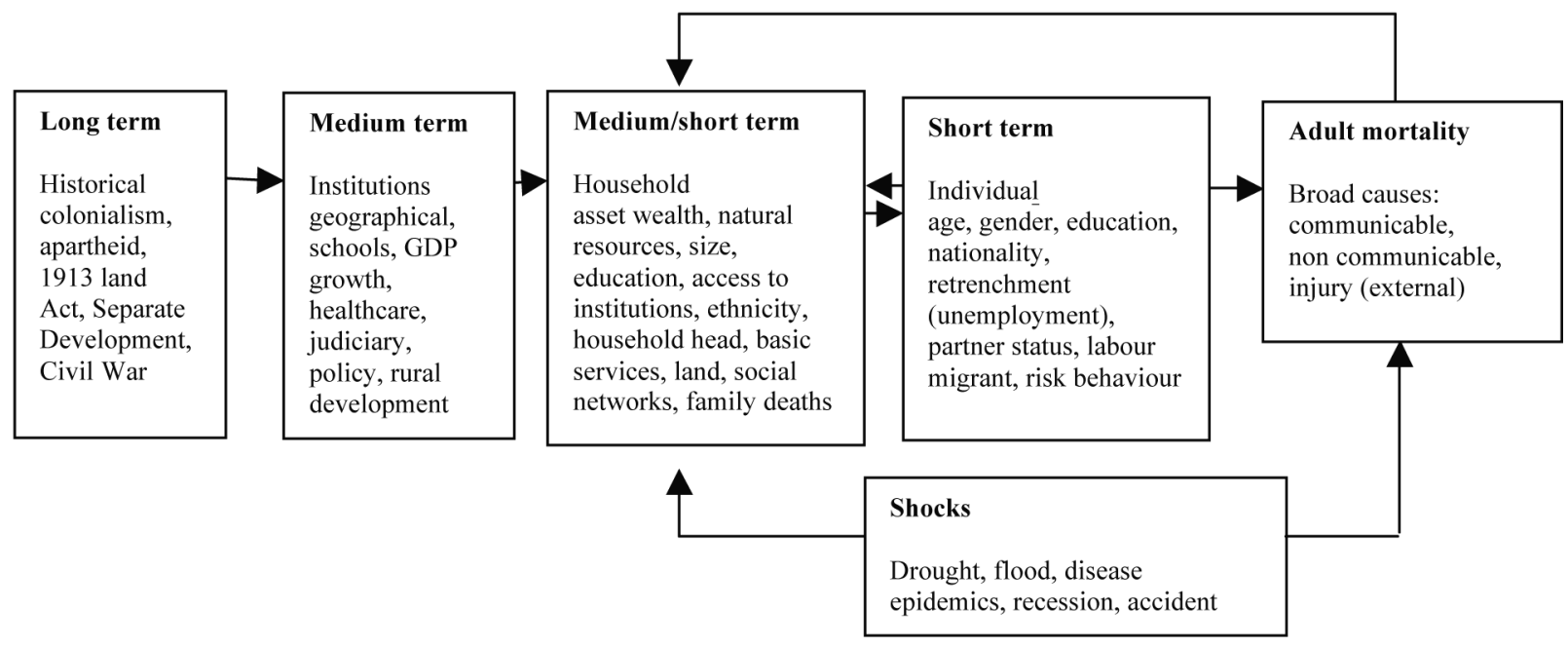

Fig. 1. The dynamics of adult mortality: a conceptual framework. 
to-event frailty modelling); and (v) highlighting potential gaps in health service delivery as a function of critical proximal threshold distance of households to nearest facility.

\section{Materials and methods}

\section{Study area and population}

The Agincourt HDSS, located in northeast South Africa (Fig. 2), was established in 1992 with annual updates of births, deaths, in- and out-migrations (Tollman et al., 1999). It is a poor, rural sub-district that includes former Mozambican refugees, temporary migrant workers and a more stable permanent population (Tollman et al., 1999). The present site covers an area of about $400 \mathrm{~km}^{2}$ and contains 25 villages and 13,500 households ${ }^{2}, 84,000$ individuals in all. An annually updated geographical information system (GIS), containing locations of all households within the site, has been established. Verbal autopsies (VA) ${ }^{3}$ were introduced in 1993. A full VA is conducted on every death recorded during the annual census update (Kahn et al., 2007). The main cause(s) of death is used in these analyses. The study population comprised all adults (15-59 years old) in the original 21 villages during the period 1993-2010. Data from four new villages added to the site since 2007 were not included in the analysis as they contribute minimal data to the study period.

\section{Outcome and explanatory variables}

The outcome in this study was defined as time (in years) contributed by an adult (15-59 years old) dur- ing the study period until right censoring ${ }^{4}(0)$ or death (1). The time to right censoring was set to either the date of permanent out-migration during the study period or as 31 December 2010 if the individual was present and alive at this endpoint. The explanatory variables included factors regarding the individual (age, gender, nationality, education, migrant pattern), the household (size, household age, death numbers, SES, household head demography, distance to nearest health facility) and the village (size, number of deaths, proportion of deaths attributed to HIV/AIDS and tuberculosis, migration patterns). A temporary migrant is defined as a labour migrant who resides in the household for less than six months but whose return is assumed, i.e. a significant link to the base household is retained (Collinson et al., 2006a). Household SES was based on living conditions, assets and services including building materials of the main dwelling, water and energy supply, ownership of modern appliances and livestock, and means of transport.

\footnotetext{
${ }^{2} \mathrm{~A}$ household is defined as a group of people who reside and eat together, plus linked temporary migrants who would eat with the household periodically.

${ }^{3}$ Verbal autopsy (VA) is a method of determining an individual's cause of death in the absence of a complete vital registration system. VAs consist of a trained interviewer using a questionnaire to collect information about the signs and symptoms of a recently deceased person from an individual familiar with the deceased. Physicians then assign the most likely cause(s) of death.

${ }^{4}$ Right censoring occurs for individuals who are still alive when they are lost to follow-up or when the study ends, i.e. a missing data problem. One makes the assumption that censoring is non-informative, i.e. event times are independent of the censoring mechanism, e.g. "missing at random".
}
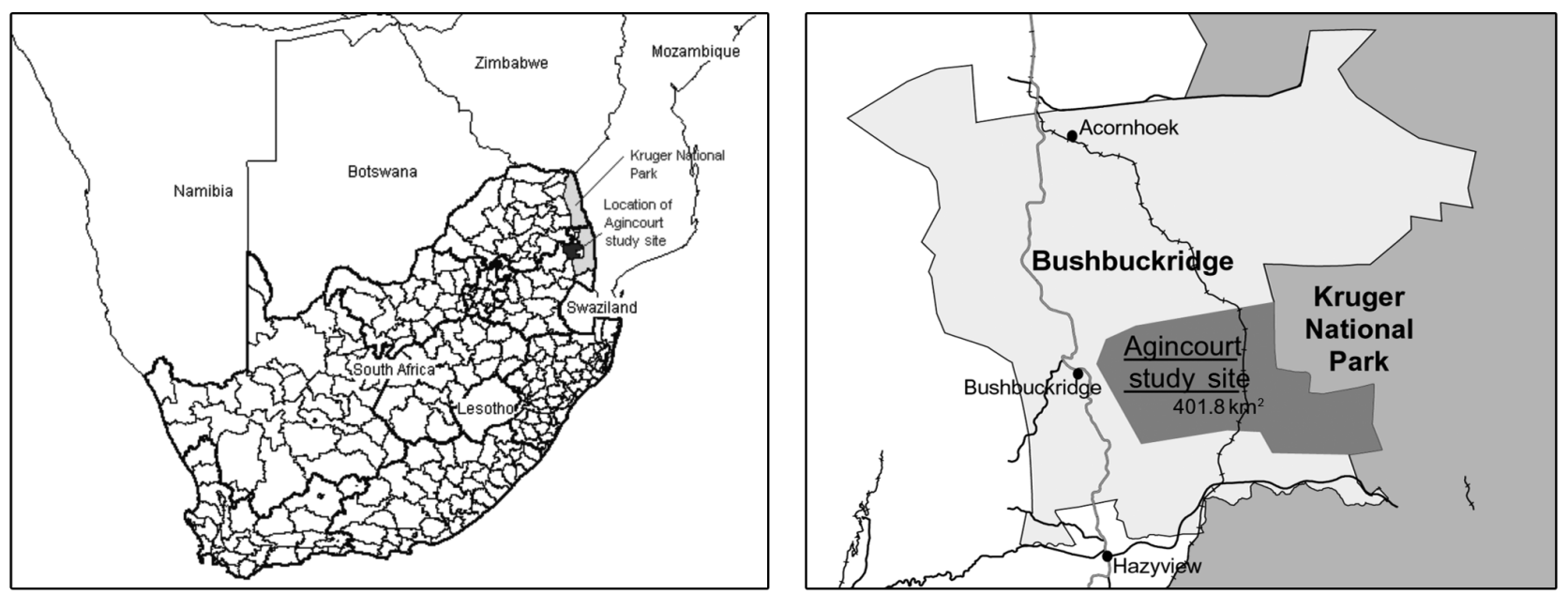

Fig. 2. Maps showing the regional location of the Agincourt HDSS. 
These assets were used to construct an SES index using multiple correspondence analysis (MCA) (Sartorius et al., 2011b).

\section{Statistical analysis}

Selected explanatory variables (e.g. education years by gender or ethnicity) were compared against one another using the standard $t$-test to better identify any potential significant underlying causal processes as suggested by risk factor analysis. A preliminary, nonparametric Cox survival analysis (Cox, 1972) was initially conducted to assess the bivariate relationship between adult mortality and each covariate. Time contributed by adults was split into discrete yearly segments to incorporate any changes in any time varying parameters such as household location. Covariates significant at the $10 \%$ level were then incorporated into the multivariable model. The assumption of proportional hazards was not upheld in the multivariable model and various parametric survival models were tested with the one providing the best fit finally adopted (the Weibull distribution (Weibull, 1951) in this case).

Given the inherent spatial and temporal correlation of longitudinal HDSS data, problems arise when using standard statistical methods as they assume independence of outcome measures, e.g. mortality. Objects in close proximity are often more alike and common exposures may influence adult mortality similarly in households of the same geographical area, introducing spatial correlation in mortality outcomes. Inclusion of the spatial effect of proximity is therefore important for efficient estimation of parameters and prediction (Wikle and Royle, 2002). Ignoring this correlation introduces bias in the risk factor analysis as the standard error of the covariates is underestimated, thereby overestimating significance. Thus, Bayesian geostatistical models are needed to analyse longitudinal spatial data as they relax assumptions of independence and assume that spatial correlation is a function of distance between locations. Bayesian approaches are being applied to the analysis of many social and health problems in addition to disease mapping and modelling or kriging (Langford et al., 1999). Their full estimation has only become possible in the last decade (Diggle et al., 1998) through software such as WinBUGS (Spiegelhalter et al., 1999) using Markov chain Monte Carlo (MCMC) simulation (Gelfand and Smith, 1990).

A Weibull Bayesian geostatistical frailty survival ${ }^{5}$ model was used to examine the multivariable association between significant covariates and adult mortali- ty. A spatial random effect at the village level, included to take account of spatial correlation, was modelled using a multivariate Gaussian distribution with a covariance matrix expressed as a parametric function of the distance between pairs of village centroid points (Diggle et al., 1998). Furthermore, an unstructured individual-level random effect was included to account for differential frailty and repeated individual observations where time episodes were split to incorporate any time varying issues such as change of household location. MCMC simulation was employed to estimate the model parameters using WinBUGS.

\section{Statistical model}

We analysed the data assuming a parametric Weibull distribution for the survivor function, where $t_{i k j}$ is the failure time of an adult $i$ (for censored observations the survival distribution is a truncated Weibull with an upper bound corresponding to the censoring time) for year $k$ at location $j$ with covariate vector $\mathrm{X}_{i k}$ and $\beta$ is a vector of unknown regression coefficients and including an unstructured individual random effect or frailty $\mathrm{h}_{i}$ and structured village-level spatial random effect $\mathrm{w}_{j}$ in the exponent of the hazard model as follows:

$$
\mathrm{t}_{i k j} \sim \operatorname{Weibull}\left(\rho, \mu_{i k j}\right) \mathrm{i}=1, \ldots, \mathrm{N}
$$

with a baseline hazard function of the form:

$$
\mathrm{l}_{0}\left(\mathrm{t}_{i k j}\right)=\rho \mathrm{t}_{i k j}^{r-1}
$$

and means for the various models as follows:

(i) multivariate non-spatial model: $\log \left(\mu_{i k j}\right)=\beta_{0}+\beta X_{i k}+h_{i}$

(ii) mutlivariate spatial model:

$$
\log \left(\mu_{i k j}\right)=\beta_{0}+\beta \mathrm{X}_{i k}+\mathrm{h}_{i}+\mathrm{w}_{j}
$$

(iii) spatial kriging model:

$$
\log \left(\mu_{i k j}\right)=\beta_{0}+\mathrm{h}_{i}+\mathrm{w}_{j}
$$

where $\beta_{0}$ is $\sim \operatorname{Normal}(0,0.1)$ and $\mathrm{w}_{j}$ has a multivariate normal distribution, $\mathrm{w}_{j} \sim \operatorname{MVN}(\underline{0}, \Sigma)$ with variance-

\footnotetext{
${ }^{5}$ Parametric survival model (assuming an underlying Weibull distribution for the survival function) incorporating a structured spatial (geostatistical) random effect and an unstructured individual-level random effect (frailty) within a Bayesian framework.
} 
covariance matrix $\Sigma$ expressed as a parametric function of distance between villages. We also assumed an isotropic stationary spatial process, where $\Sigma m n=\sigma_{w}^{2}$ $\exp \left(-\varphi d_{m n}\right), d_{m n}$ is the Euclidean distance between villages $m$ and $n, \sigma_{w}^{2}$ is the geographical variability known as the sill, $\varphi$ is a smoothing parameter that controls the rate of correlation decay with increasing distance and measures the range of geographical dependency (Diggle et al., 1998). We specified $\varphi$ as a uniform distribution between $\varphi_{\min }$ and $\varphi_{\max }$ (Gelfand and Vounatsou, 2003). The range was defined as the minimum distance at which spatial correlation between locations is below $5 \%$. This distance can be calculated as $3 / \varphi$ meters. The individual-level frailty was assumed to follow an independent normal distribution $\mathrm{h}_{i} \sim$ $\mathrm{N}\left(0, \sigma_{b}^{2}\right)$. Vague Normal distributions were adopted for the $\beta$, inverse gamma priors for the variance parameters and a uniform prior for $\rho$. The shape parameter of the Weibull survival distribution $(\rho)$ was given a non-informative gamma distribution with a mean and variance of 1 .

MCMC simulation was applied to fit the models. We ran a single chain sampler with a burn-in of 5,000 iterations. Convergence was assessed by running the simulation until the Monte Carlo error for each parameter of interest was less than $5 \%$ of the sample standard deviation. The chains thereafter were sampled every single iteration until a sample size of 10,000 had been attained.

\section{Model assessment}

The analysis was carried out in STATA, version 12.0 SE (Stata Corp., 2007) and in WinBUGS (Spiegelhalter et al., 1999). Model comparison in STATA was based on the Akaike information criterion (AIC). We selected the model with smallest value of AIC and then graphically examined the model fit in STATA using Cox-Snell residual plots. The Deviance information criterion (DIC) was used to assess the various multivariate models (Spiegelhalter et al., 2002). Generally, the smaller the AIC/DIC value, a measure of the relative goodness-of-fit of a statistical model, the better the fit.

\section{Population-attributable fractions (PAR)}

The PAR approach is needed as measure that an effect does not factor in the prevalence of exposure to selected determinants in a population and thus allows identification of relevant exposures for targeting by policy makers at the population level. The following standard formula for calculating PAR for each determinant is based on the proportion of the total population exposed to the risk factor $\left(p_{e}\right)$ factoring in the multivariable-adjusted model risk coefficient (HR):

$$
\mathrm{PAR}=\frac{p_{e}(\mathrm{HR}-1)}{1+p_{e}(\mathrm{HR}-1)}
$$

\section{Risk maps}

Simulation-based Bayesian kriging (Gelfand et al., 1999) at regular grid points was used to produce smoothed maps of all-cause and cause-specific adult mortality risk within the study area. Apart from a constant, these models included only the random effects. All identifying features, e.g. village centroids and village boundaries, were removed from the maps to ensure confidentiality and avoid potential stigmatisation of high-risk villages. Also, the HIV/AIDS and tuberculosis mortality risk map is not shown for this reason. Model estimates were exponentiated to relative risks. A simple map showing potential high-risk areas as a function of the straight-line distance to nearest health facility was constructed using a circular buffer zone around health facilities based on the significant cut-off found in the bivariate risk factor analysis. The spatial risk maps were constructed in MapInfo Professional version 9.5 (MapInfo, 2008).

\section{Results}

\section{Descriptive analysis}

Between 1993 and 2010, there were 104,969 adults (15-60 years old) and 5,675 adult deaths within the 21 villages. The mean age at entry was 24.6 years (standard deviation (SD) 11) and the majority were female $(59,781$ or $57 \%$ ). Of those whose nationality was known, 33,192 or $32 \%$ were of Mozambican origin with the remainder mostly being South African. The average level of education, as expressed by the number of years at school completed, was 6.2 (SD 4.5) with this mean being significantly higher in males compared to females (6.29 versus $6.06, \mathrm{P}<0.001)$. Similarly, the mean number of years attained by South Africans in general was significantly higher than that of their Mozambican counterparts (7.14 versus 4.30, $\mathrm{P}<0.001)$. Males, on average, were significantly more likely to spend fewer resident months within the site each year compared to females (mean of 9.76 versus $7.98, \mathrm{P}<0.001$ ). This figure for South African adults in general was also lower compared to Mozambicans (8.93 versus 9.01, $\mathrm{P}<0.001$ ). 
Main cause of death

Of the 5,675 adult deaths that occurred from 1993 up to the end of 2010, the majority were attributed to communicable causes $(2,989$ or $53 \%)$, largely attributed to HIV/AIDS and tuberculosis, with 2,527 deaths or $45 \%$ (95\% confidence interval (CI), 43.7-46.3\%). Non-communicable diseases were responsible for 1,262 or $22.2 \%$ of all adult deaths over the study period. Approximately 11\% (643) adult deaths were attributed to external causes. Mozambican adults had a significantly higher proportion of deaths attributed to communicable causes ( $58 \%$ versus $51 \%$, P <0.001). Conversely, Mozambicans had a significantly lower proportion of deaths attributed to non-communicable disease $(17 \%$ versus $24 \%, \mathrm{P}<0.001)$. There was no significant difference in the proportion of adults deaths attributed to external causes by nationality $(\mathrm{P}=$ $0.303)$. Females had a significantly higher proportion of deaths attributed to communicable causes (56\% versus $50 \%, \mathrm{P}<0.001)$ and a significantly higher proportion of deaths attributed to non-communicable causes $(25 \%$ versus $20 \%, \mathrm{P}<0.001)$. Males did, however, have a much higher proportion of deaths attributed to external causes $(17 \%$ versus $5 \%, \mathrm{P}<0.001)$.

\section{Temporal trend analysis}

During the period 1993-2010, the overall adult mortality rate was 8.2 (95\% CI, 8.0-8.4) adult deaths per 1,000 person-years. Mortality significantly ( $\mathrm{P}<0.001)$ increased over the study period, with the most pronounced increase occurring from 1999 onwards to reach a plateau of $\sim 13$ deaths per 1,000 person-years in 2004 with similar levels observed

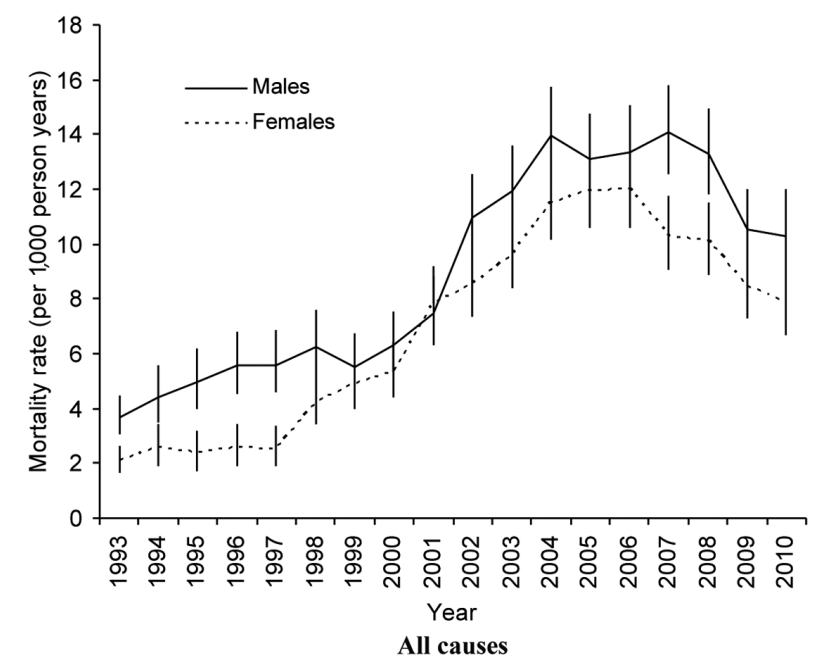

through to 2007, after which we observed a sharp decline in adult mortality (Fig. 3). Males generally had a higher mortality rate except for a convergence that occurred around 2001, during the upsurge in mortality related communicable disease, largely HIV/AIDS and tuberculosis (Fig. 1). A concurrent (though at a lower level) rise in non-communicable disease mortality occurred over the 1993-2004 period with a decline thereafter. Levels of external mortality remained fairly constant over the study period.

\section{Risk factor analysis and impact}

Increasing the time period (1999-2004 and 20052010 versus 1993-1998) was found to be a significant risk factor for adult mortality and confirms the temporal trend (Table 1) seen in Fig. 3. The most prominent and significant individual level risk factors for adult mortality following multivariable adjustment were younger adult age (15-44 years), male gender, partner having died, low level of education, migrant status and unemployment (Table 1). Conversely partner being alive was found to be significantly protective when compared to the baseline group of never married. No significant difference in adult mortality risk by nationality (former Mozambican versus South African) was identified $(\mathrm{P}=0.592)$ and was thus not included in the final multivariable model. Similarly, no significant difference in mortality risk was identified when comparing older (aged $\geq 45$ years) adult Mozambicans to older South Africans.

Following multivariable adjustment, householdlevel determinants that remained significantly associated with adult mortality were: increasing number of other adult deaths in the household, residing in a

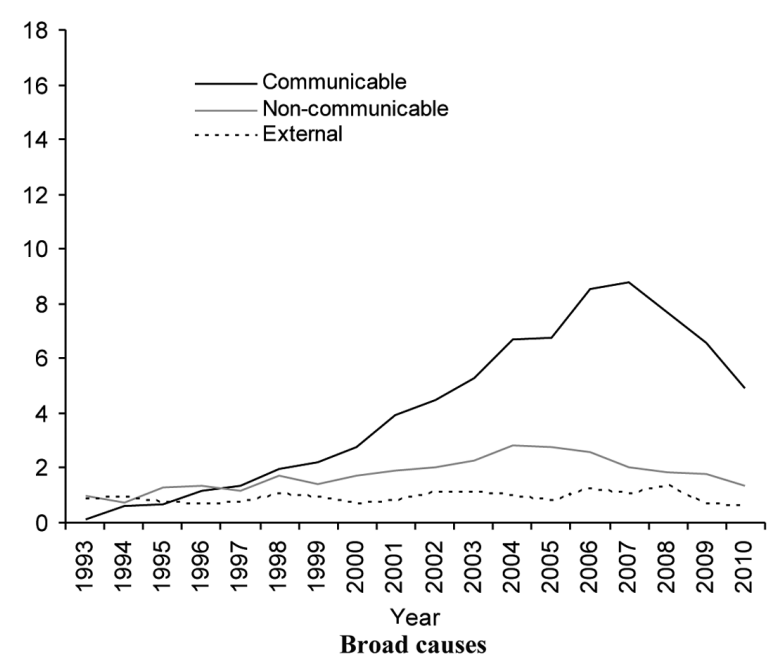

Fig. 3. Annual adult (15-59) mortality rates per 1,000 person-years by cause, Agincourt sub-district, South Africa, $1993-2010$. 


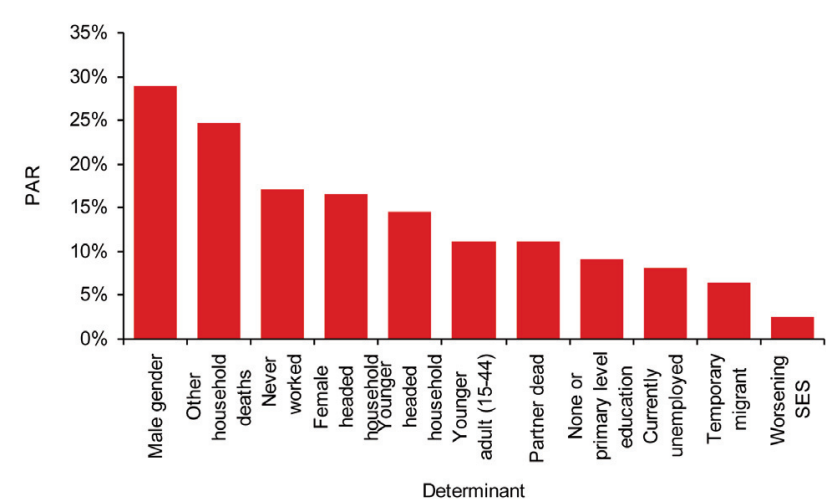

Fig. 4. Descending population attributable fractions (PAR) of high impact determinants found to be significantly associated with adult mortality following multivariable adjustment, Agincourt, 1993-2010.

household either headed by a female, a younger adult ( $<40$ years of age) or where the household head had died (Table 1). Worsening SES status was also found to be a significant risk factor for increased adult mortality risk. Large distance to a health facility was found to be significantly associated with adult mortality risk at a bivariate level. This was, however, not statistically significant following multivariable adjustment. Increased distance from a main road was found to be a significant risk factor for adult mortality and, conversely, significantly protective with regard to injuryrelated (external) mortality (Table 1 ).

At the village or community level, villages with a lower proportion of deaths attributed to HIV/AIDS and tuberculosis (proxy for prevalence) were found to have a significantly lower risk of adult mortality based on bivariate association. This was no longer significant following multivariable adjustment. No significant relationship was identified between more mobile (migrant) communities or villages and adult mortality (Table 1). The most attributable determinant was male gender, followed by other household adult deaths, unemployment, female and younger household head followed by partner death (Table 1, Fig. 4). Other relevant exposures can be seen in Table 1 and Fig. 2.

\section{Spatial risk maps of adult mortality}

Five distinct foci of higher adult mortality can be seen in Fig. 5. Three are in the central to upper central region of the site and two in the south-east. These mainly correlate to areas with higher risk of communicable disease mortality, largely HIV/AIDS and tuberculosis. Higher, non-communicable disease mortality risk was observed in five distinct foci in the upper-central, south-east and western parts of the site (Fig. 5). The strong clustering of injury-related (external) mortality was evident in the central region of the site and in an area in the western part. Based on the circular buffer zones around health facilities (relating threshold straight-line distance to a health facility seen in risk factor analysis), we see that households in five villages in particular appear to have a higher mortality
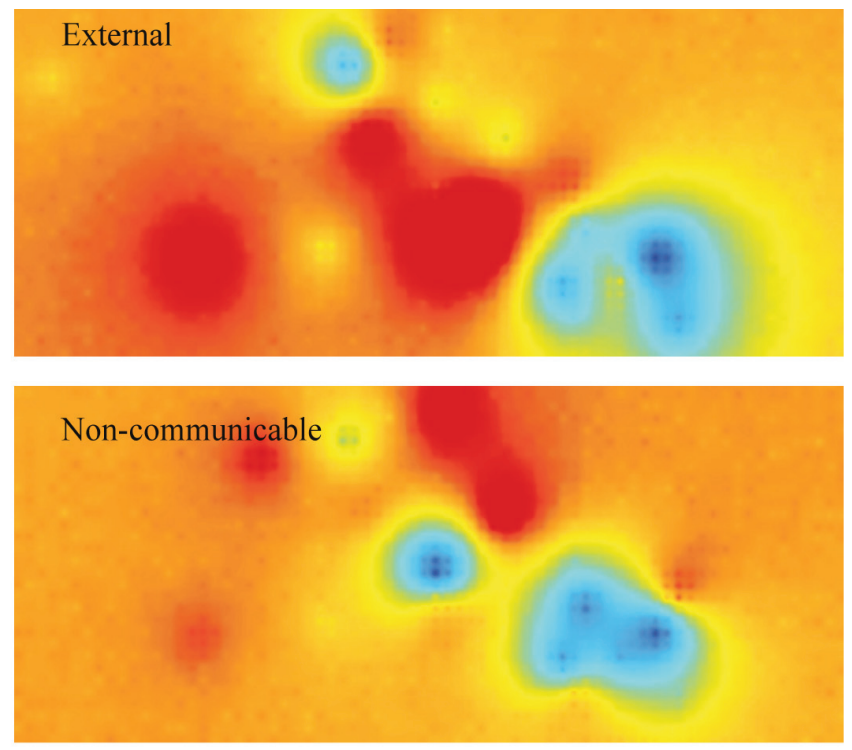

$\geq 1.20$ $1.05-1.19$ $0.95-1.04$ $0.50-0.94$

Fig. 5. Spatial relative risk (RR) of broad cause-specific adult mortality based on prediction estimates from Bayesian spatial kriging models, Agincourt sub-district, 1993-2010. 
Table 1. Bivariate and multivariable analysis of risk factors for adult mortality (15-59 years), Agincourt sub-district, $1993-2010$.

\begin{tabular}{|c|c|c|c|c|c|c|}
\hline Risk factors & Bivariate $\mathrm{n}$ & $\operatorname{HR}(95 \% \mathrm{CI})$ & P-value & $\begin{array}{c}\text { Multivariable } \\
\text { HR }(95 \% \text { BCI) }\end{array}$ & P-value & $\mathrm{PAR}^{\mathrm{a}}$ \\
\hline \multicolumn{7}{|l|}{ Temporal } \\
\hline 1993-1998 & 53,752 & 1.00 & & 1.00 & & \\
\hline $1999-2004$ & 62,002 & $2.19(2.02,2.37)$ & $<0.001$ & $4.83(4.22,5.53)$ & $<0.001$ & - \\
\hline $2005-2010$ & 64,835 & $2.84(2.63,3.07)$ & $<0.001$ & $6.35(5.49,7.35)$ & $<0.001$ & - \\
\hline \multicolumn{7}{|l|}{ Individual factors } \\
\hline Age (45-60 versus $15-44$ age group) & $17,102 / 97,568$ & $0.61(0.56,0.66)$ & $<0.001$ & $0.53(0.48,0.58)$ & $<0.001$ & $11.2 \%$ \\
\hline Male gender & 44,637 & $1.36(1.29,1.43)$ & $<0.001$ & $1.86(1.74,1.99)$ & $<0.001$ & $28.9 \%$ \\
\hline Mozambican nationality & 32,910 & $1.02(0.96,1.08)$ & 0.592 & - & - & - \\
\hline \multicolumn{7}{|l|}{ Partner status } \\
\hline Never married & 46,848 & $1.05(0.98,1.12)$ & 0.163 & 1.00 & & \\
\hline Partner alive & 51,206 & $0.74(0.69,0.79)$ & $<0.001$ & $0.75(0.70,0.81)$ & $<0.001$ & $13.3 \%$ \\
\hline Partner dead & 5,424 & $2.12(1.96,2.30)$ & $<0.001$ & $2.76(2.52,3.02)$ & $<0.001$ & $11.2 \%$ \\
\hline \multicolumn{7}{|l|}{ Education status } \\
\hline Secondary or higher level education & 57,314 & 1.00 & & 1.00 & & \\
\hline None or primary level education & 31,702 & $1.24(1.17,1.32)$ & $<0.001$ & $1.28(1.2,1.38)$ & $<0.001$ & $9.1 \%$ \\
\hline \multicolumn{7}{|l|}{ Last employment status } \\
\hline Employed & 24,524 & 1.00 & & 1.00 & & \\
\hline Not employed & 9,172 & $1.60(1.48,1.73)$ & $<0.001$ & $1.70(1.57,1.84)$ & $<0.001$ & $8.2 \%$ \\
\hline Never worked & 36,640 & $1.40(1.30,1.51)$ & $<0.001$ & $1.61(1.48,1.75)$ & $<0.001$ & $17.0 \%$ \\
\hline Unknown & 33,142 & $3.94(3.68,4.22)$ & $<0.001$ & $7.99(7.33,8.71)$ & $<0.001$ & - \\
\hline Temporary migrant $^{\mathrm{b}}$ & 97,477 & $1.10(1.04,1.16)$ & 0.001 & $1.17(1.10,1.25)$ & $<0.001$ & $6.4 \%$ \\
\hline \multicolumn{7}{|l|}{ Household level factors } \\
\hline Household size & 103,329 & $1.00(0.99,1.01)$ & 0.821 & - & - & - \\
\hline \multicolumn{7}{|l|}{ Average education status of adult occupants } \\
\hline Secondary or higher level education & 57,583 & 1.00 & & \multirow{2}{*}{\multicolumn{3}{|c|}{$\begin{array}{l}\text { Not included as correlated with } \\
\text { individual education status }\end{array}$}} \\
\hline None or primary level education & 45,895 & $1.20(1.13,1.26)$ & $<0.001$ & & & \\
\hline \multicolumn{7}{|l|}{ Any other adult household deaths } \\
\hline None & 100,911 & 1.00 & & 1.00 & & \\
\hline $1+$ & 985 & $59.9(53.4,67.2)$ & $<0.001$ & $34.61(30.6,39.2)$ & $<0.001$ & $24.7 \%$ \\
\hline Female household head & 103,190 & $1.61(1.53,1.70)$ & $<0.001$ & $1.53(1.44,1.63)$ & $<0.001$ & $16.6 \%$ \\
\hline Older household head, aged $\geq 40$ years & 103,190 & $1.47(1.39,1.57)$ & $<0.001$ & $1.65(1.55,1.77)$ & $<0.001$ & $14.5 \%$ \\
\hline Mozambican household head & 102,606 & $1.00(0.94,1.06)$ & 0.942 & - & - & - \\
\hline Household head death & 103,478 & $1.46(1.33,1.61)$ & $<0.001$ & $1.21(1.09,1.34)$ & $<0.001$ & $1.4 \%$ \\
\hline \multicolumn{7}{|l|}{ Maximum household SES tertiale } \\
\hline Least poor & 10,940 & 1.00 & & 1.00 & & \\
\hline Poor & 11,197 & $1.27(1.17,1.38)$ & $<0.001$ & $1.11(1.02,1.21)$ & 0.013 & $2.0 \%$ \\
\hline Most poor & 10,741 & $1.57(1.45,1.69)$ & $<0.001$ & $1.17(1.07,1.27)$ & $<0.001$ & $3.0 \%$ \\
\hline Unknown & 70,600 & $0.6(0.56,0.65)$ & $<0.001$ & $0.60(0.53,0.67)$ & $<0.001$ & - \\
\hline \multicolumn{7}{|l|}{ Euclidian distance to nearest health care facility } \\
\hline$<5 \mathrm{~km}$ & 97,116 & 1.00 & & 1.00 & & \\
\hline$\geq 5 \mathrm{~km}$ & 3,726 & $1.14(1.00,1.30)$ & 0.056 & $1.03(0.89,1.18)$ & 0.690 & $0.1 \%$ \\
\hline Increasing distance $(\mathrm{km})$ from road (all-cause) & 103,478 & $1.03(1.00,1.07)$ & 0.064 & - & - & - \\
\hline Increasing distance $(\mathrm{km})$ from road (HIV/TB) & 103,478 & $1.07(1.01,1.12)$ & 0.017 & - & - & - \\
\hline Increasing distance $(\mathrm{km})$ from road (external) & 103,478 & $0.86(0.77,0.97)$ & 0.010 & - & - & - \\
\hline \multicolumn{7}{|l|}{ Community (village) level factors } \\
\hline Average adult migrant months per village and year & 97,477 & $1.02(0.97,1.07)$ & 0.530 & - & - & - \\
\hline Proportion of adult deaths due to HIV/TB $(<40 \%)$ & 103,478 & $0.92(0.85,0.99)$ & 0.050 & $0.97(0.89,1.06)$ & 0.529 & $0.4 \%$ \\
\hline
\end{tabular}

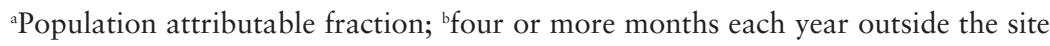


risk as a function of increased distance to the nearest local primary health care clinic (Fig. 6). These specific areas are numbered in this figure with many households falling outside the risk catchment zone in location 2, particularly in the upper-central region of the site.

\section{Discussion}

\section{Trends and leading causes of death}

A substantial and significant increase in adult mortality was observed between 1999 and 2006, which is in line with other estimates for South Africa (Dorrington et al., 2001; Hosegood et al., 2004). HIV/AIDS and tuberculosis was the leading cause-ofdeath among adults $(\sim 45 \%)$ and largely drove the observed temporal pattern. This is comparable to other estimates for SSA in 2001 where HIV/AIDS was estimated to account for $40-45 \%$ of adult mortality (Mathers et al., 2006). The levelling out of adult mortality could possibly be linked to the antiretroviral therapy (ART) rollout which began in South Africa round 2004. Non-communicable mortality is prevalent despite the larger pronounced impact of communicable disease (HIV/AIDS and tuberculosis) mortality (Tollman et al., 2008). Non-communicable disease mortality accounted for approximately $22 \%$ of all adult deaths in our study population over this period, which is in line with estimates of $20 \%$ for SSA
(Mathers et al., 2006). Thus, while communicable disease mortality remains high, indicating a relatively early stage of the epidemiological transition, increases in non-communicable disease mortality indicates the need for preparedness to deal with this potential double burden.

\section{Spatial hotpots}

The spatial hotspots of increased adult mortality risk, largely driven by communicable diseases, were observed in the upper-central and south-eastern regions of the Agincourt sub-district. This distribution is due to a complex web of interacting factors that include increased communicable disease mortality (HIV/AIDS and tuberculosis) and non-communicable mortality, in particular in high-risk areas. Lifestylerelated mortality among more affluent (South African) households may be driving the observed non-communicable mortality risk in the upper-central and western parts of the sub-district. However, whatever the cause, the risk maps (Fig. 5.) can be used for the evaluation, design and implementation of relevant interventions, e.g. ART rollout. Further research into the underlying reasons for higher risk among older adult South Africans relative to Mozambicans is needed to better inform relevant policy and interventions.

Male gender was found to be a prominent risk factor in this study and this has been demonstrated on numerous occasions (Mathers et al., 2006; WHO,

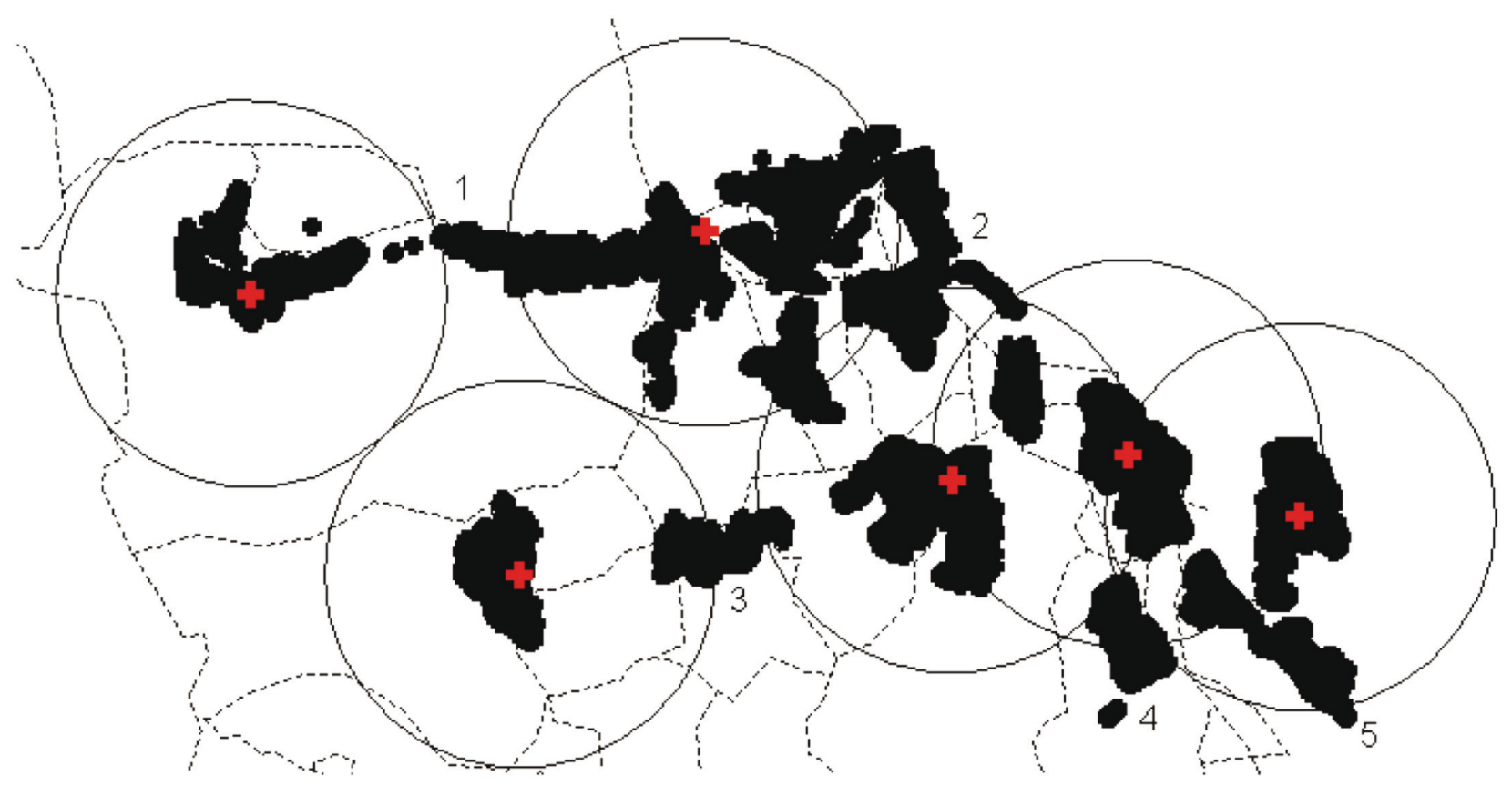

Fig. 6. Household $(\bullet)$, road $(---)$ and health facility $(+)$ locations with concentric circles showing the $5 \mathrm{~km}$ capture zone of these health facilities, Agincourt sub-district. Note: potentially higher risk household areas discussed above are numbered 1-5. 
2006) due to, for example, inherent biological differences and a tendency for males to engage in higher risk activities such as alcohol consumption (Ezzati et al., 2002). Males have also been shown to be more likely to be labour migrants than females (Lurie, 2000; Collinson et al., 2006a). We also observed a significantly higher mortality risk in younger to middle-aged adults (15-44 years) compared to older adults (45-59 years), likely due to HIV. This determinant was also found to have a relatively high attributable fraction, thus suggesting a high impact in this population. It is likely that an underlying interplay of higher-risk behaviour, sexual activity (exposure to HIV) and mobility (migration) is driving this observed age-risk differential (Lurie, 2000; Ezzati et al., 2002; Collinson et al., 2006a). Education has been previously associated with improved adult survival in other African settings (Berhane et al., 2002). We have similarly demonstrated that no, or only primary level, education was a significant and high-impact predictor of adult mortality in Agincourt. Worsening SES or poverty seems to be an important determinant of adult mortality in Agincourt as has been demonstrated in other African settings (Fantahun et al., 2008). Poor SES and high unemployment have led to external migration for work, while low education levels may explain false perceptions of HIV exposure risk; over $90 \%$ of men perceived little or no personal risk (Collinson et al., 2006b). A previous study in Agincourt has shown that long-distance migrants, who returned once or twice a year, report having many partners, leading to increased risk for their partners in Agincourt (Collinson et al., 2006b). This has all led to an explosive spread of HIV in this and other rural settings. We observed a strong and high impact association between partner status and adult mortality risk. Strategies that enable more frequent contact between migrant men and their rural families are urgently needed, as are HIV prevention and the need to raise awareness of this risk amongst all men in this area (Collinson et al., 2006b).

Mozambican nationality was not a risk factor for adult mortality. Previous studies in this area have shown an increased risk of mortality among Mozambican children in the age group of the 1-5 years old (Hargreaves et al., 2004; Sartorius et al., 2011a), suggesting a normalisation of risk in adulthood. This is likely due to the adjustment for lower education and SES in the model (which was more evident among the Mozambicans) and as a result of these settlements being further away from health facilities. The risk for adult mortality was higher in the poorest villages, sug- gesting that the benefits of improved health care are not evenly distributed throughout the study area.

Female-headed households, those headed by males or females younger than 40 years, or other adult household arrangements appeared to be the highest impact household-level determinants. Other adult household deaths as a risk factor for adult mortality is in line with previous findings (Sartorius et al., 2010, 2011a). This in turn impacts SES through loss of working-age adults. Cost of funerals and the loss of income (impacting household SES) are also some of the reasons explaining this phenomenon (Collins and Leibbrandt, 2007). Direct transmission of HIV between unreported partnerships may also explain a more direct link to other adult household deaths. A cycle of greater male deaths (see above), leading to more female headed households is likely. The pronounced impact of HIV/AIDS and tuberculosis in this setting is also likely to have reduced the age of household heads as a result of previous mortality of those heading households. Given that a male household head conferred a survival advantage to adults (also shown in previous studies for infants and children (Sartorius et al., 2010, 2011a) and that male migrants are at increased risk, could potentially compound adult mortality over time as household head dynamics change. This cycle coupled with the direct and indirect impacts of adult mortality on children and the elderly is leading to vulnerable households that should be targeted for social support (Feachem et al., 1992). Female-headed households also need to be targeted as they appear to be more vulnerable and at higher risk for child, adult and overall mortality.

Adult populations in mortality hotspots likely experienced differential access to health care as a large distance from the nearest health facility emerged as a significant risk factor in the bivariate analysis. This association has been shown in other studies on adult mortality (Ali et al., 2007) and specifically with regards to ART access in South Africa (Ingle et al., 2010). This study found that with in service access need to be addressed. Critical distance threshold catchment maps for facilities can further highlight areas at increased risk of adult mortality for policy makers.

In this study we also observed an increased risk of adult mortality in households closer to main roads. A recent study in rural Kwazulu-Natal found significant HIV prevalence clustering near a national road and a strong exponential decrease in HIV prevalence with increasing distance from this road (Tanser et al., 2009). Individuals living in communities with better access to transport and transport routes may be at 
higher risk of HIV infection (Arroyo et al., 2006; Ferguson and Morris, 2007; Tanser et al., 2009). Our study thus confirms that risks for HIV (mortality) are associated with specific, socio-geographical locations, which would allow targeting of tailored education and behavioural interventions in these areas.

Villages with a high fraction of adult deaths attributed to HIV/AIDS and tuberculosis (a crude proxy for the underlying prevalence) were also found to be a significant risk factor at the bivariate level. However, following multivariable adjustment this was no longer significant, suggesting that more proximal household level determinants may be more important.

Despite major improvements in vital registration in South Africa, cause of death data still suffer from potential underreporting, "ill-defined" over-classification and misclassification, especially with regards to HIV/AIDS and injury-related mortality (Bradshaw et al., 2010; Groenewald et al., 2010). HDSS implementing physician-coded verbal autopsies are thus often the only way to assess cause-specific mortality fractions and trends (Kaufman et al., 1997; Kuh and Shlomo, 2004). However, physician-coded VAs have known limitations, such as relying heavily on household recall of medical records and related information, which affects its applicability in low-resource settings (Murray et al., 2007). Misclassification of data could thus have occurred with respect to our study, especially with regard to underestimating non-specific HIV/AIDS-related and non-communicable disease. However, a previous validation study of VAs in Agincourt has shown that it performs well in this high-HIV prevalence setting (Kahn et al., 2000). Other studies have also confirmed that VA data can be used to reasonably estimate the distribution of AIDS- and non-AIDS-related deaths, even in a rural population with relatively low levels of education (Doctor and Weinreb, 2003).

\section{Conclusions}

Despite HDSS not necessarily being representative of national patterns, given the validity of our findings in relation to other studies, identified trends in this setting may reflect the level and changing nature of adult mortality in rural South Africa.

This study demonstrates the use of advanced methods to:

(i) pinpoint high risk areas for adult mortality and that variation is present even in a small geographical area; and

(ii) correctly estimate and identify high impact risk factors.
PAR is extremely important for policy makers as it provides additional information above strength of association in that one can estimate the reduction of an outcome if a given risk factor is removed. This gives the policy maker the sense of were resources might be most effectively allocated or targetted in resource-poor settings. Health programmes need to take this into account when assessing and further planning the comprehensive plan to tackle adult mortality in this and other rural populations.

\section{Acknowledgements}

This work was supported by a PhD fellowship from the South African Centre for Epidemiological Modelling and Analysis (SACEMA), a National Research Foundation (NRF) Centre of Excellence, as well as research fellowships from the MRC/Wits Rural Public Health and Health Transitions Research Unit (Agincourt) through the Wellcome Trust (grant no. 069683/Z/02/Z), UK, and the Swiss-South Africa Joint Research Programme (project no. JRP IZLSZ3_122926). Additional funding was provided by a Wits Faculty of Health Sciences Medical Research Endowment Fund (MREF) (grant no. SARB000). The Agincourt HDSS was funded by the Wellcome Trust, UK (grant no. 058893/Z/99/A, 069683/Z/02/Z, 069683/Z/08/Z), the University of the Witwatersrand, the South African Medical Research Council, and the Andrew W. Mellon Foundation, USA.

\section{References}

Ali M, Jin Y, Kim D, De Z, Park J, Ochiai R, Dong B, Clemens J, Acosta C, 2007. Spatial risk for gender-specific adult mortality in an area of southern China. Int J Health Geogr 6, 31. Arroyo MA, Sateren WB, Serwadda D, Gray RH, Wawer MJ, Sewankambo NK, Kiwanuka N, Kigozi G, Wabwire-Mangen F, Eller M, Eller LA, Birx DL, Robb ML, McCutchan FE, 2006. Higher HIV-1 incidence and genetic complexity along main roads in Rakai District, Uganda. J Acq Immun Def Synd 43, 440-445.

Berhane Y, Hogberg U, Byass P, Wall S, 2002. Gender, literacy, and survival among Ethiopian adults, 1987-96. Bull World Health Organ 80, 714-720.

Bradshaw D, Pillay-Van Wyk V, Laubscher R, Nojilana B, Groenewald P, Nannan N, Metcalf C, 2010. Cause of death statistics for South Africa: challenges and possibilities for improvement. South African MRC Burden of Disease Research Unit.

Brown AF, Ettner SL, Piette J, Weinberger M, Gregg E, Shapiro MF, Karter AJ, Safford M, Waitzfelder B, Prata PA, Beckles GL, 2004. Socioeconomic position and health among persons with diabetes mellitus: a conceptual framework and review of 
the literature. Epidemiol Rev 26, 63-77.

Bryceson DF, 2004. Agrarian vista or vortex: African rural livelihood policies. Rev Afr Pol Econ 31, 617-629.

Collins D, Leibbrandt M, 2007. The financial impact of HIV/AIDS on poor households in South Africa. AIDS 21, S75S81.

Collinson M, Tollman S, Kahn K, Clark S, 2006a. Highly prevalent circular migration: households, mobility and economic status in rural South Africa. Johannesburg: Wits University Press.

Collinson M, Wolff B, Tollman S, Kahn K, 2006b. Trends in internal labour migration from the rural Limpopo province, male risk behaviour, and implications for spread of HIV/AIDS in rural South Africa. J Ethn Migr Stud 32, 633-648.

Cox DR, 1972. Regression models and life-tables. J R Stat Soc 187-220.

Diggle P, Moyeed R, Tawn J, 1998. Model-based geostatistics (with discussion). Appl Stat 48, 299-350.

Doctor H, Weinreb A, 2003. Estimation of AIDS adult mortality by verbal autopsy in rural Malawi. AIDS 17, 2509-2513.

Dorrington R, Bourne D, Bradshaw D, Laubscher R, Timæus I, 2001. The impact of HIV/AIDS on adult mortality in South Africa. MRC Technical Report. South Africa: Burden of Disease Unit.

Ezzati M, Lopez A, Rodgers A, Vander Hoorn S, Murray C, and the Comparative Risk Assessment Collaborating Group, 2002. Selected major risk factors and global and regional burden of disease. Lancet 360, 1347-1360.

Fantahun M, Berhane Y, Hogberg U, Wall S, Byass P, 2008. Young adult and middle age mortality in Butajira demographic surveillance site, Ethiopia: lifestyle, gender and household economy. BMC Public Health 8, 268.

Feachem R, Philips M, Bulatao R, 1992. Introducing adult health. In: The health of adults in the developing world. Feachem R, Kjellstrom T, Murray C, Over M, Phillips M (eds). New York: Oxford University Press.

Ferguson A, Morris C, 2007. Mapping transactional sex on the Northern Corridor highway in Kenya. Health Place 13, 504519.

Gelfand A, Ravishanker N, Ecker M, 1999. Modeling and inference for point-referenced binary spatial data: Marcel Dekker Inc.

Gelfand A, Smith A, 1990. Sampling-based approaches to calculating marginal densities. J Am Stat Assoc 85, 398-409.

Gelfand A, Vounatsou P, 2003. Proper multivariate conditional autoregressive models for spatial data analysis. Biostatistics 4, 11-25.

Groenewald P, Msemburi W, Neethling I, Day C, TuoaneNkhazi M, Bradshaw D, 2010. Burden of Disease. In: Day C, Barron P, Massyn N, Padarath A, English R. (eds.) District Health Barometer 11.

Hargreaves J, Collinson M, Kahn K, Clark S, Tollman S, 2004.
Childhood mortality among former Mozambican refugees and their hosts in rural South Africa. Int J Epidemiol 33, 12711278.

Hofstede G, 1986. Cultural differences in teaching and learning. Int J Intercult Rel 10, 301-320.

Hosegood V, Vanneste A, Timaeus I, 2004. Levels and causes of adult mortality in rural South Africa: the impact of AIDS. AIDS 18, 663-671.

Ingle S, May M, Uebel K, Timmerman V, Kotze E, Bachmann M, Sterne J, Egger M, Fairall L, for the International Epidemiologic Databases to Evaluate AIDS Southern Africa (IeDEA-SA), 2010. Differences in access and patient outcomes across antiretroviral treatment clinics in the Free State province: a prospective cohort study. S Afr Med J 100, 675-681.

Kahn K, Tollman S, Collinson M, Clark S, Twine R, Clarke B, Shabangu M, Gomez-Olive F, Mokoena O, Garenne M, 2007. Research into health, population and social transitions in rural South Africa: data and methods of the Agincourt health and demographic surveillance system. Scand J Public Health 69, 820.

Kahn K, Tollman S, Garenne M, Gear J, 2000. Validation and application of verbal autopsies in a rural area of South Africa. Trop Med Int Health 5, 824-831.

Kaufman J, Asuzu M, Rotimi C, Johnson O, Owoaje E, Cooper R, 1997. The absence of adult mortality data for sub-Saharan Africa: a practical solution. Bull World Health Organ 75, 389395.

Kuh D, Shlomo Y, 2004. A life course approach to chronic disease epidemiology: Oxford University Press.

Langford I, Leyland A, Rashbas J, Goldstein H, 1999. Multilevel modeling of the geographic distribution of diseases. J Roy Stat Soc C-App 48, 253-268.

Lurie M, 2000. Migration and AIDS in Southern Africa: a review. S Afr J Sci 96.

Machethe CL, 2004. Agriculture and poverty in South Africa: can agriculture reduce poverty? Overcoming underdevelopment Pretoria.

Mathers C, Lopez A, Murray C, 2006. The burden of disease and mortality by condition: data, methods and results for 2001. In: Global burden of disease and risk factors. Lopez AD, Mathers CD, Ezzati M, Jamison D, Murray CJL (eds). New York: Oxford University Press, 45-240 pp.

Mertz O, Wadley R, Christensen A, 2005. Local land use strategies in a globalizing world: subsistence farming, cash crops and income diversification. Agr Syst 85, 209-215.

Murray C, Feachem R, 1990. Adult mortality in developing countries. Trans R Soc Trop Med Hyg 84, 1-2.

Murray C, Lopez A, Feehan D, Peter S, Yang G, 2007. Validation of the symptom pattern method for analyzing verbal autopsy data. PLoS Med 4, e327.

Murray CJL, Lopez AD, 1997. Mortality by cause for eight regions of the world: global burden of disease study. Lancet 
349, 1269-1276.

Preston S, Nelson V, 1974. Structure and change in causes of death: an international summary. Pop Stud 28, 19-51.

Sartorius B, Kahn K, Vounatsou P, Collinson M, Tollman S, 2010. Young and vulnerable: spatial-temporal trends and risk factors for infant mortality in rural South Africa (Agincourt), 1992-2007. BMC Public Health 10, 645.

Sartorius B, Kahn K, Vounatsou P, Collinson M, Tollman S, 2011a. Survived infancy but still vulnerable: spatial-temporal trends and risk factors for child (1-4) mortality in rural South Africa (Agincourt), 1992-2007. Geospat Health 5, 285-295.

Sartorius K, Sartorius B, Tollman S, Schatz E, Kirsten J, Collinson M, 2011b. Rural poverty dynamics and refugee communities in South Africa: a spatial-temporal model. Popul Space Place 19, 103-123.

Schatz E, Ogenmefun C, 2007. Caring and contributing: the role of older women in multi generational households in the HIV/AIDS era. World Dev 35, 1390-1403.

Schwarze S, Zeller M, 2005. Income diversification of rural households in Central Sulawesi, Indonesia. Q J Int Agr 44, 6173.

Sherbinin A, Van Wey W, McSweeny K, Aggarwal R, Barbieri A, Henry S, Hunter L, Twine W, Walker R, 2008. Rural household demographics, livelihoods and the environment. Global Environ Change 18, 38-53.

Spiegelhalter D, Best N, Carlin B, van der Linde A, 2002. Bayesian measures of model complexity and fit. J Roy Stat Soc B 64 583-639.

Spiegelhalter D, Thomas A, Best N, 1999. WinBUGS version 1.2 user manual MRC Biostatistics Unit.

Tanser F, Barnighausen T, Cooke G, Newell M, 2009. Localized spatial clustering of HIV infections in a widely disseminated rural South African epidemic. Int J Epidemiol 38, 1008-1016. Tollman S, Herbst K, Garenne M, Gear J, Kahn K, 1999. The Agincourt demographic and health study-site description, baseline findings and implications. S Afr Med J 89, 858-864.

Tollman S, Kahn K, Sartorius B, Collinson M, Clark S, Garenne M, 2008. Implications of mortality transition for primary health care in rural South Africa: a population-based surveillance study. Lancet 372, 893-901.

Vermeulen S, Nawir A, Mayers J, 2008. Rural poverty reduction through business partnerships? Examples of experience from the forestry sector. Environ Dev Sustain 10, 1-18.

Victora CG, Huttly SR, Fuchs SC, Olinto MT, 1997. The role of conceptual frameworks in epidemiological analysis: a hierarchical approach. Int J Epidemiol 26, 224-227.

Weibull W, 1951. A statistical distribution function of wide applicability. J Appl Mech 18, 293-297.

WHO, 2002. The world health report 2002: reducing risks, promoting health life. Geneva: World Health Organization.

WHO, 2006. The world health report 2006: working together for health. Geneva: World Health Organization.

WHO, 2008. Indicator definitions and metadata. WHO Statistical Information - System - WHOSIS 2008; Geneva.

Wikle C, Royle J, 2002. Spatial statistical modelling in biology. Encyclopaedia of Life Support Systems. EOLSS Publishers Co. Ltd.

Williamson O, 2000. The new institutional economics: taking stock, looking ahead. J Econ Lit 27, 595-613.

Xiangxing S, Zhong Z, Shuguang H, Chong S, 2008. A review of the major projects constituting the China Academic Digital Library. Electron Libr 26, 39-54. 